\title{
Bi-modal control of vacuum-powered soft pneumatic actuators with embedded liquid metal-based strain sensitive skin
}

\author{
Matthew A. Robertson ${ }^{1}$, Student Member, IEEE, Laurent Dejace ${ }^{2}$, \\ Stéphanie P. Lacour ${ }^{2}$, Member, IEEE and Jamie Paik ${ }^{1}$, Member, IEEE
}

\begin{abstract}
Soft robotic systems are composed of active and passively deformable structures which are intrinsically compliant, flexible, and elastic. Although these features offer benefits of adaptability, robustness, and safety, controlling these types of robots is a significant challenge, in part from the difficulty of obtaining feedback from sensors which provide state information without hindering the advantageous material properties which grant these systems their unique mechanical behavior. We demonstrate here the first integration of a flexible, stretchable, liquid metal-based strain sensor with vacuumpowered soft pneumatic actuators (V-SPAs) for simultaneous controlled feedback of the soft actuators as well as user input and soft robotic device interaction. The soft sensors which are encapsulated within a Polydimethylsiloxane (PDMS) membrane are directly embedded in the outer body skin of the soft actuators, and can be used to correlate the deformation of the body under vacuum actuation to overall actuator strain or to detect external disturbances. This information is used to compute and control the angle of a rotational 3-DoF actuator module, as well as detect implicit user input control signals by direct interaction without the need for an external control interface. The dual use of embedded sensing shown in this work provides a fundamental strategy for soft collaborative robot applications.
\end{abstract}

Index Terms-Soft pneumatic actuators, stretchable sensors, soft robotics

\section{INTRODUCTION}

Soft robots are designed to leverage benefits that many biological organisms are known to exploit for achieving compliance, robustness, and adaptability to their environment [1]. These features have been described as the basis for what has been termed morphological computation [2], [3], allowing the mechanical properties of the constitutive soft body tissues of the living systems to passively conform to objects around them without invoking complex control to do so. This effectively reduces the problem in tasks such as manipulation or locomotion [4], [5] but does not completely eliminate the need for higher level control [6].

In soft robotics the issue of control is at the front of current challenges in the field. Although the best approach to this problem has not been settled, primarily between modelbased and model free control methods [7]-[9], a widely accepted starting point is the acknowledged need for implicit

\footnotetext{
${ }^{1}$ Reconfigurable Robotics Laboratory, Institute of Mechanical Engineering, School of Engineering, École Polytechnique Fédérale de Lausanne, Lausanne (Switzerland). Correspondance:jamie.paik@epfl.ch

${ }^{2}$ Laboratory for Soft Bioelectronic Interfaces (LSBI), Institutes of Microengineering, Institute of Bioengineering, Centre for Neuroprosthetics, École Polytechnique Fédérale de Lausanne (EPFL), 1202 Geneva (Switzerland).
}
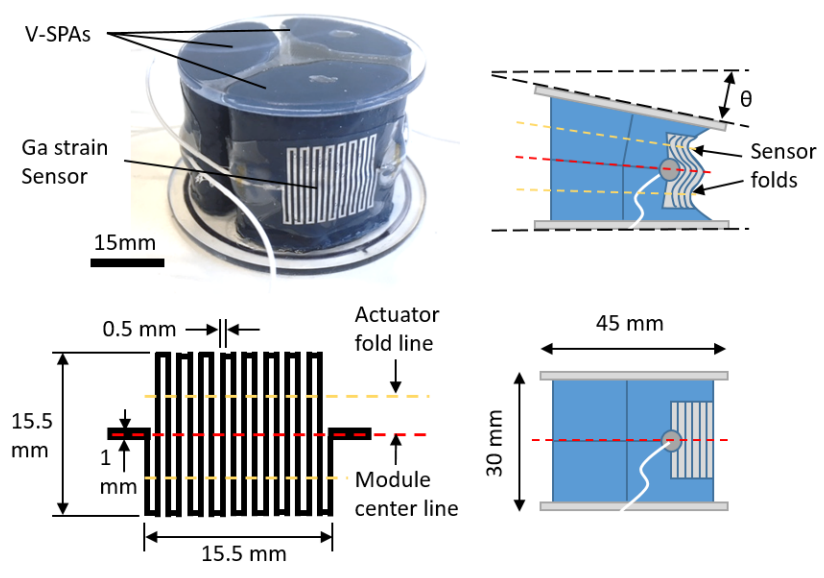

Fig. 1. Sensorized V-SPA module. A soft, liquid metal strain sensor integrated with a 3-DoF vacuum-powered soft pneumatic actuator module enables sensing of its angular deformation upon activation. A single sensor is bonded to each actuator in the module and comprised of a meandering path which is positioned at the centerline of the actuator. This pattern extends above and below the two longitudinal locations where folds occur due to buckling when activated.

sensing strategies. The baseline requisite for sensors which are compatible with soft structures makes even this starting point a challenge.

In order to preserve the benefits of soft material robot body and actuator compliance, any sensing system used in a fully integrated soft robotic system should be likewise compliant. While most commonly available robotic sensors are rigid, various types of soft, flexible, and stretchable sensors have been investigated in research toward developing soft robots which are innervated with sensing abilities to interact with the environment [10], [11] as well as provide feedback for control. The sensing principles utilized in previous work varies between resistive, capacitive, and optic types [12], [13].

Among the diverse soft sensor types that have been explored, liquid metal based sensors achieve some of the highest performance in terms of stretchability, accuracy, and highly thin form factor [14], [15]. This type of sensor also stands out from the perspective of fabrication, as some can be readily printed by direct writing methods while others can be produced using a variety of versatile deposition techniques, allowing application to wearable devices or soft robotic structures and actuators [16]-[20]. The low-profile 
form factor and exceptional softness of these sensors have even made them suitable for use in medical and implantable applications [21], [22]. In comparable efforts, roboticists have also integrated these sensors directly into soft actuators and robotic bodies to provide proprioceptive feedback to control grasping force and detect objects for gripping [23], [24].

Although liquid metal type sensors show diverse advantages and applications, these have primarily been utilized for modes of deformation which induce strain of the sensor. This mode has made integration of these sensors with positive pressure soft pneumatic actuators (SPAs) readily feasible, however, recently studied SPAs powered by vacuum (VSPAs) do not operate in the same way [25]-[27]. Rather than stretching from inflation of soft elastomeric chambers, V-SPAs generate force and motion primarily from buckling and folding of flexible but not necessarily elastic encasement. Although the dominant mode of this type of actuator does not directly match the main functional mode for many soft sensors, the versatility of liquid metal-based sensors enables output when used in either stretching or compression mode, making them a preferred candidate for use with vacuum powered actuators. The benefits afforded by V-SPAs include enhanced failsafe behavior since they do not rely on elastic deformation and thus do not store energy while active, as well as their being power limited since their force output relies on environmental pressure conditions which are generally held constant. These features thus offer new opportunities to explore for unique integrated actuator and sensing systems for the benefit of extending versatility and safety in soft robotics as a whole.

\section{SOFT ROBOTIC SYSTEM HARDWARE}

\section{A. V-SPA modules}

Actuators are fabricated individually on preform structures, with two open-cell polyurethane foam body chambers, and a single central dividing plane, as described in previous work [25]. When activated by vacuum depressurization, this rigid membrane component (heavy gauge paper) helps to enforce buckling primarily in the vertical direction. The soft foam actuator cores are coated with two manually brushed layers of Elastosil M4601 mixed with a blue color additive for improved visual contrast, to become sealed actuator structures. Circular acrylic plates are laser cut for the top and bottom module structures, between which three V-SPAs are glued using Loctite 406 adhesive, formulated specifically for silicone rubbers. Before the actuators are separated from their preforms and acrylic plates are attached to form a 3-DoF rotational module, a strain sensor is first bonded to the outer surface of each as described in the subsequent fabrication steps.

\section{B. Liquid metal sensor skin}

The actuator skin sensor is first fabricated independently from the actuator on a PDMS membrane substrate using microfabrication processes to produce an exceptionally thin sensor structure. Similar types of sensors have been achieved

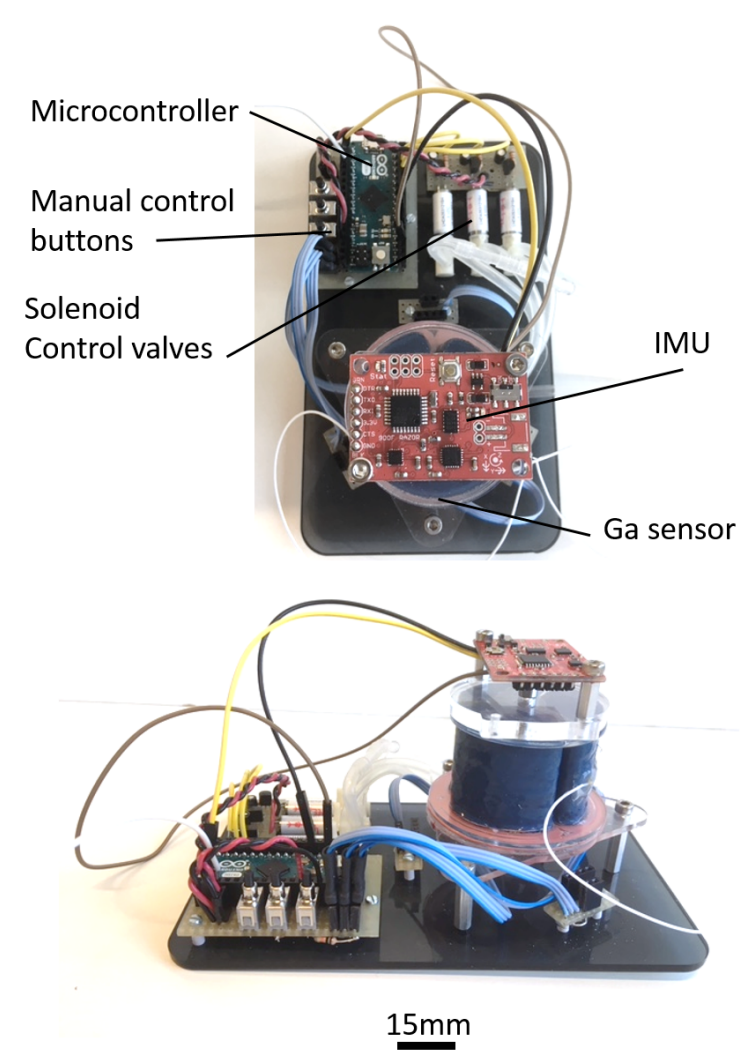

Fig. 2. Pneumatic control platform. A control board was constructed and utilized for performing characterization and control testing.

by other fabrication techniques, including liquid metal injection, however the resulting metallization in these sensors are an order of magnitude thicker in other cases than the process used here. The substrate is prepared with an array of microscale pillars fabricated by soft lithography, and precoated with a thin film of sputtered gold $(\mathrm{Au})$ to enhance chemical affinity of $\mathrm{Ga}$ to the substrate layer. These features act to uniformly collect and distribute vapor deposited $\mathrm{Ga}$ with controlled thickness $(1.5 \mu \mathrm{m})$ into continuous thin film liquid metal patterns. Once the electrically conductive patterns have been rendered, a top layer of PDMS is applied to enclose the liquid metal traces within a thin membrane sensor structure, $150 \mu \mathrm{m}$ in thickness [28]. Although the resulting liquid metal features are highly stretchable with low electrical resistance, the electromechanical coupling (gauge factor) of Ga is low (roughly 1), such that meanders are needed to increase the cumulative resistance change produced by sensor strain which in turn increases the sensor's read-out signal strength. The winding pattern chosen for integration with the V-SPAs can be seen in Figure 1 The conductive traces are positioned to span across the direction of actuator folding and buckling induced by vacuum activation. This ensures the most traces of the sensor are subjected to compressive deformation at the inside of a fold, consequently resulting in high electrical sensitivity to actuator strain. As the connected traces are deformed in compression, the total resistance of the 
liquid metal pathway is reduced. This change in resistance is measured using a voltage divider connected to a 10-bit analog input pin of a microcontroller, with a $2.5 \mathrm{~V}$ reference.

\section{Actuator-Sensor integration}

The V-SPAs and liquid metal skin sensors are combined using a plasma bonding technique. The outer surface of the actuators and back side of the thin sensors are chemically activated by 30 seconds of exposure to oxygen plasma in a sealed vacuum chamber, and then the two active surfaces subsequently bonded upon contact with each other. The parameters used for plasma generation were $10 \mathrm{~W}$ with an $\mathrm{O}_{2}$ pressure of 0.5 mbar. The free ends of the sensor traces are positioned at the middle of the winding pathway to coincide with the position of the rigid dividing membrane of the actuator, since this location undergoes the least deformation during activation, and is therefore ideal for the placement of wires attached to the sensor. Thin sensor output wires are attached to the liquid metal traces with silicone sealant for mechanical strain relief.

\section{Multi-module robotic arm platform}

Two sensorized, 3-DoF V-SPA modules were produced and stacked in series to form a soft robotic arm with embedded sensing capabilities. With three actuators, each module can produce rotational tilting motion through activation of different combinations of the individual actuators. The two complete modules are arranged inline, such that each actuator of a module is situated above one from the module below. The pneumatic vacuum source is then shared and controlled together along one axial side of the robot arm, for the sake of simplicity, although future implementations and studies could just as easily operate each actuator independently to allow more total degrees of freedom. The arm is built onto a pneumatic control board (shown in Figure 2) which contains a microcontroller and three solenoid valves each connected to one linear side of the soft robot arm. The valves can be controlled either in feedback control mode dictated by the embedded sensors and microcontroller programs, or through direct operation from three manual button switches also built into the board. While the manual switches only allow binary control of the V-SPAs in each module, a PWM signal from the microcontroller can be used to activate the valves at high frequency to simulate regulated pressure. The PWM control method is used to control the module angle, where varying the duty cycle produces variable angle output.

\section{EXPERIMENTS}

\section{A. Embedded sensor characterization}

A single sensorized actuator module was characterized to correlate the embedded sensor measurements to the final output angle of a 3-DoF module. An inertial measurement unit (IMU) was attached to the top plate of a V-SPA module, and both the measurements from the IMU and the voltage output of one embedded strain sensor were connected to the microcrontoller. While logging both sensor readings, a series of ten binary step inputs were commanded to the

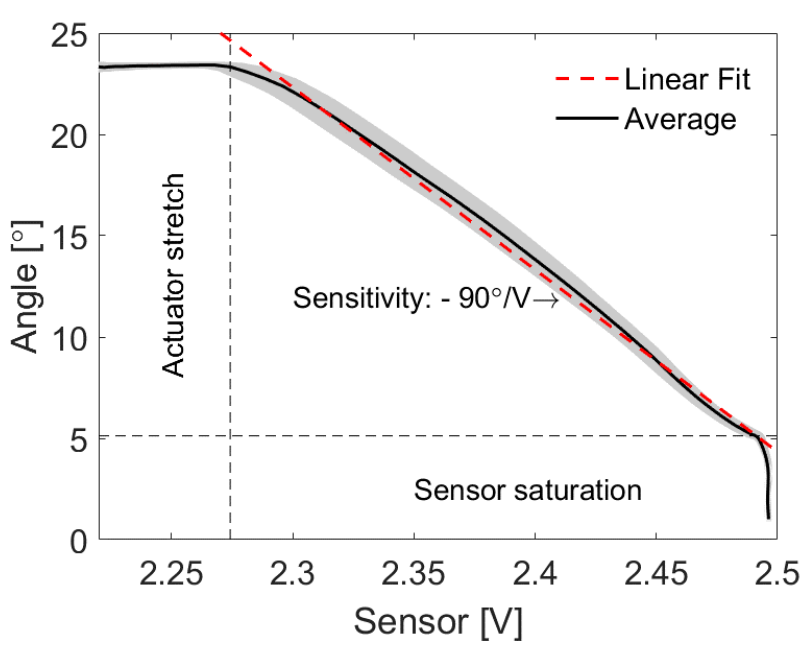

Fig. 3. Strain sensor characterization. The sensitivity of the Ga sensor integrated with the actuator of a V-SPA module is shown with a dominantly linear relationship between sensor output, and actuator module angle.

actuator with the combined strain sensor at $85.1 \%$ vacuum. These ten steps were divided and averaged together for both sensor readings, and the resulting measurements plotted against each other to obtain the plot shown in Figure 3. This figure shows the relationship between the voltage output of the embedded strain sensor and output angle of a V-SPA module is generally linear, which allows the calculation of a sensitivity factor from a first order fit, of approximately $-90 / \mathrm{V}$, where

$$
\text { Module angle }=-90 * \text { sensor voltage. }
$$

The plot also shows two discontinuous effects near the ends of the actuator displacement caused mostly likely by actuator and sensor stretching near the maximum module displacement, and sensor saturation at low displacement (higher sensor voltage output). The total measured range of the soft Ga sensor is seen to be within roughly $0.5 \mathrm{~V}$, which was measured with over 100 bit resolution using the analog signal microcontroller inputs.

\section{B. Closed loop control}

Using the sensitivity factor derived from the characterization experiment, we implemented a proportional closedloop angular position controller for a sensorized V-SPA module. A series of step input targets were prescribed and the integrated soft sensor was used to provide feedback to track the set points, spaced at 10, 15, and 20 degrees. In order to achieve variable displacement, the pressure and flow to the tested actuator was required to be variable. Since the solenoid valves used are not proportional, a pulse width modulated (PWM) duty cycle was used to drive the valves, and achieve and approximately proportional pressure regulation. The frequency of the duty cycle was set to 30 Hz. Although the measured angular deflection of the module followed the set points closely, fluctuations were observed around these targets as a result of driving the valves in an 


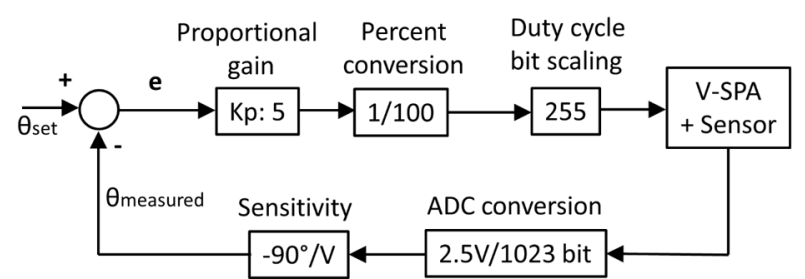

(a)

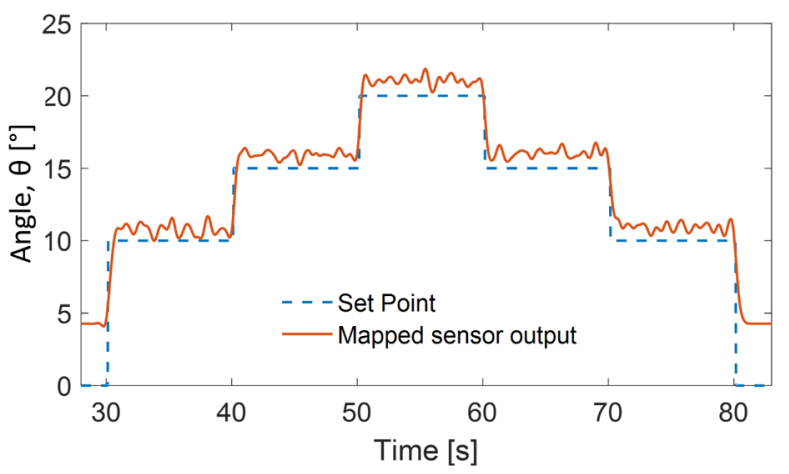

(b)

Fig. 4. Closed loop sensorized V-SPA angle step tracking. (a) Block diagram of the control scheme (b) The angular trajectory of V-SPA module is shown relative to a prescribed set point target. An offset in the sensor reading is observed before and after the initial and final steps, but this is below the threshold of sensitivity as shown from initial characterization study.

intermittent manner. This effect was partially attenuated by the inclusion of a flow restricting membrane with a small hole between the vacuum supply from the valve and the actuator itself. This passive element introduced a small delay between valve activation and actuator motion, negligible for relatively lower frequency actuator motions but effective for the higher frequency supply line fluid oscillations. The block diagram for this control scheme and the resulting performance of the position tracker is shown in Figure 4.

\section{COllaborative ROBOT CONTROL}

The multi-module robotic arm was tested to demonstrate an alternative utilization of the embedded actuator skin sensors, as human user input detection. In this tested scenario, no peripheral user controls are needed to direct the motion of the robotic arm. Rather, a more intuitive form of commands are issued by direct contact with the soft robotic structure, similar to the instruction which may be given between humans in supervised motion learning tasks. In the robotic case, similar hands-on motion teaching is feasible as illustrated by the simple demonstration shown in Figure 5. This capability leverages the embedded sensory skin combined with the V-SPA modules, which can then in turn also be used to carry out the directed motions under closed loop control, either in response to continued human user interaction, or to follow primitive programmed motions or behaviors.

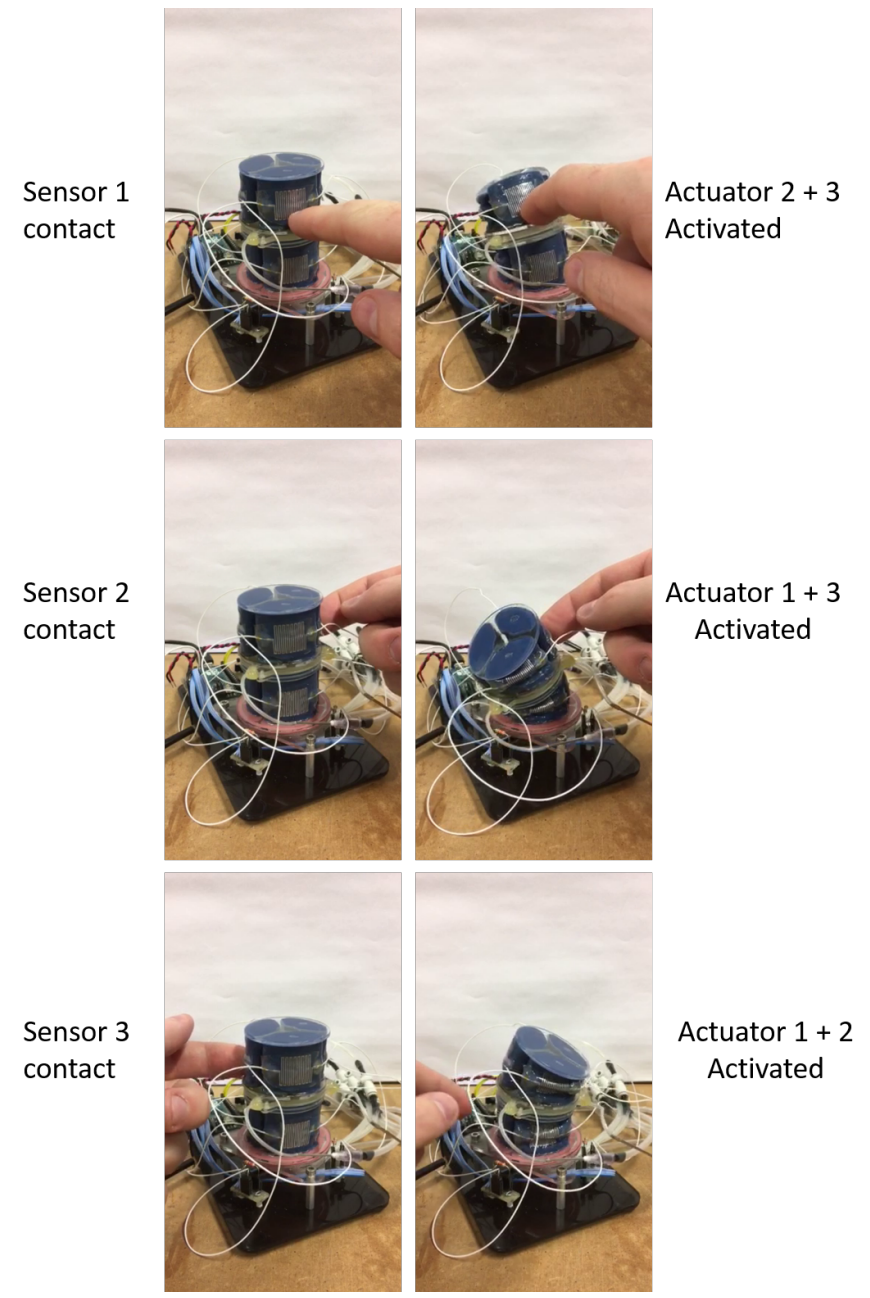

Fig. 5. Implicit user control. Embedded soft sensors enable intuitive, direct manual control of a two module soft robotic arm.

\section{Discussion}

In this work we demonstrated the first integration and validation of soft liquid metal Ga sensors with V-SPAs, for measuring and controlling large actuator strain. The mode of deformation unique to V-SPAs includes primarily buckling and folding, which is complimented by the compliance and ability of liquid metal-based sensors to deform without impeding the natural motion of the soft actuator. This compatibility gives rise to measurable resistance changes due to the compression of the actuator and embedded sensor structure. We validated the capability of localized deformations induced by V-SPA activation to be sufficient for generating useful sensor output signals for both control and user interaction, which are critical features of soft robotic systems that are particularly well suited operation in close proximity to humans in the framework of collaborative applications.

\section{ACKNOWLEDGMENT}

This work was supported by the Swiss National Science Foundation (SNSF), the National Centre of Competence in 
Research (NCCR) Robotics (Switzerland) and the Fondation Bertarelli.

\section{REFERENCES}

[1] S. Kim, C. Laschi, and B. Trimmer, "Soft robotics: a bioinspired evolution in robotics," Trends in Biotechnology, vol. 31, no. 5, pp. 287-294, May 2013. [Online]. Available: http://www.sciencedirect.com/science/article/pii/S0167779913000632

[2] R. Pfeifer and J. C. Bongard, How the Body Shapes the Way We Think: A New View of Intelligence (Bradford Books). The MIT Press, 2006.

[3] K. Ghazi-Zahedi, R. Deimel, G. Montfar, V. Wall, and O. Brock, "Morphological computation: The good, the bad, and the ugly," in 2017 IEEE/RSJ International Conference on Intelligent Robots and Systems (IROS), Sep. 2017, pp. 464-469.

[4] R. Deimel and O. Brock, "A novel type of compliant and underactuated robotic hand for dexterous grasping," The International Journal of Robotics Research, vol. 35, no. 1-3, pp. 161-185, Jan. 2016. [Online]. Available: https://doi.org/10.1177/0278364915592961

[5] C. Paul, "Morphological computation: A basis for the analysis of morphology and control requirements," Robotics and Autonomous Systems, vol. 54, no. 8, pp. 619-630, Aug. 2006. [Online]. Available: http://www.sciencedirect.com/science/article/pii/S0921889006000613

[6] N. Farrow, Y. Li, and N. Correll, "Morphological and Embedded Computation in a Self-contained Soft Robotic Hand," arXiv:1605.00354 [cs], May 2016, arXiv: 1605.00354. [Online]. Available: http://arxiv.org/abs/1605.00354

[7] D. Rus and M. T. Tolley, "Design, fabrication and control of soft robots," Nature, vol. 521, no. 7553, pp. 467-475, May 2015.

[8] V. Vikas, P. Grover, and B. Trimmer, "Model-free control framework for multi-limb soft robots," in 2015 IEEE/RSJ International Conference on Intelligent Robots and Systems (IROS), Sep. 2015, pp. 11111116.

[9] C. Duriez, "Control of elastic soft robots based on real-time finite element method," in 2013 IEEE International Conference on Robotics and Automation, May 2013, pp. 3982-3987.

[10] H. Zhao, K. OBrien, S. Li, and R. F. Shepherd, "Optoelectronically innervated soft prosthetic hand via stretchable optical waveguides," Science Robotics, vol. 1, no. 1, p. eaai7529, Dec. 2016. [Online]. Available: http://robotics.sciencemag.org/content/1/1/eaai7529

[11] J. C. Yeo, H. K. Yap, W. Xi, Z. Wang, C.-H. Yeow, and C. T. Lim, "Flexible and Stretchable Strain Sensing Actuator for Wearable Soft Robotic Applications," Advanced Materials Technologies, vol. 1, no. 3, p. 1600018, Jun. 2016. [Online]. Available: https://onlinelibrary.wiley.com/doi/abs/10.1002/admt.201600018

[12] M. Amjadi, K.-U. Kyung, I. Park, and M. Sitti, "Stretchable, Skin-Mountable, and Wearable Strain Sensors and Their Potential Applications: A Review," Advanced Functional Materials, vol. 26, no. 11, pp. 1678-1698, Mar. 2016. [Online]. Available: https://onlinelibrary.wiley.com/doi/abs/10.1002/adfm.201504755

[13] E. L. White, M. C. Yuen, J. C. Case, and R. K. Kramer, "Low-Cost, Facile, and Scalable Manufacturing of Capacitive Sensors for Soft Systems," Advanced Materials Technologies, vol. 2, no. 9, p. 1700072, Sep. 2017. [Online]. Available: https://onlinelibrary.wiley.com/doi/abs/10.1002/admt.201700072

[14] R. K. Kramer, C. Majidi, R. Sahai, and R. J. Wood, "Soft curvature sensors for joint angle proprioception," in 2011 IEEE/RSJ International Conference on Intelligent Robots and Systems, Sep. 2011, pp. 1919-1926.

[15] H. O. Michaud, L. Dejace, S. d. Mulatier, and S. P. Lacour, "Design and functional evaluation of an epidermal strain sensing system for hand tracking," in 2016 IEEE/RSJ International Conference on Intelligent Robots and Systems (IROS), Oct. 2016, pp. 3186-3191.

[16] J. W. Boley, E. L. White, G. T.-C. Chiu, and R. K. Kramer, "Direct Writing of Gallium-Indium Alloy for Stretchable Electronics," Advanced Functional Materials, vol. 24, no. 23, pp. 3501-3507, Jun. 2014. [Online]. Available: https://onlinelibrary.wiley.com/doi/abs/10.1002/adfm.201303220

[17] M. G. Mohammed and R. Kramer, "All-Printed Flexible and Stretchable Electronics," Advanced Materials, vol. 29, no. 19, p. 1604965, May 2017. [Online]. Available: https://onlinelibrary.wiley.com/doi/abs/10.1002/adma.201604965
[18] R. L. Truby, M. Wehner, A. K. Grosskopf, D. M. Vogt, S. G. M. Uzel, R. J. Wood, and J. A. Lewis, "Soft Somatosensitive Actuators via Embedded 3d Printing," Advanced Materials, vol. 30, no. 15, p. 1706383, Apr. 2018. [Online]. Available: https://onlinelibrary.wiley.com/doi/abs/10.1002/adma.201706383

[19] A. Hirsch, L. Dejace, H. O. Michaud, and S. P. Lacour, "Harnessing the Rheological Properties of Liquid Metals To Shape Soft Electronic Conductors for Wearable Applications," Accounts of Chemical Research, Feb. 2019. [Online]. Available: https://doi.org/10.1021/acs.accounts.8b00489

[20] X. Wang, R. Guo, and J. Liu, "Liquid Metal Based Soft Robotics: Materials, Designs, and Applications," Advanced Materials Technologies, vol. 4, no. 2, Feb. 2019. [Online]. Available: https://onlinelibrary.wiley.com/doi/abs/10.1002/admt.201800549

[21] R. Herbert, J.-H. Kim, Y. Kim, H. Lee, W.-H. Yeo, R. Herbert, J.-H. Kim, Y. S. Kim, H. M. Lee, and W.-H. Yeo, "Soft Material-Enabled, Flexible Hybrid Electronics for Medicine, Healthcare, and HumanMachine Interfaces," Materials, vol. 11, no. 2, p. 187, Jan. 2018 [Online]. Available: https://www.mdpi.com/1996-1944/11/2/187

[22] S. P. Lacour, G. Courtine, and J. Guck, "Materials and technologies for soft implantable neuroprostheses," Nature Reviews Materials, vol. 1 , no. 10, p. 16063 , Oct. 2016. [Online]. Available: https://www.nature.com/articles/natrevmats201663

[23] N. Lu and D.-H. Kim, "Flexible and Stretchable Electronics Paving the Way for Soft Robotics," Soft Robotics, vol. 1, no. 1, pp. 53-62, Jul. 2013. [Online]. Available: https://www.liebertpub.com/doi/abs/10.1089/soro.2013.0005

[24] R. A. Bilodeau, E. L. White, and R. K. Kramer, "Monolithic fabrication of sensors and actuators in a soft robotic gripper," in 2015 IEEE/RSJ International Conference on Intelligent Robots and Systems (IROS), Sep. 2015, pp. 2324-2329.

[25] M. A. Robertson and J. Paik, "New soft robots really suck: Vacuum-powered systems empower diverse capabilities," Science Robotics, vol. 2, no. 9, 2017. [Online]. Available: http://robotics.sciencemag.org/content/2/9/eaan6357

[26] D. Yang, M. S. Verma, E. Lossner, D. Stothers, and G. M. Whitesides, "Negative-Pressure Soft Linear Actuator with a Mechanical Advantage," Advanced Materials Technologies, vol. 2, no. 1, p. 1600164, Oct. 2016. [Online]. Available: https://onlinelibrary.wiley.com/doi/abs/10.1002/admt.201600164

[27] D. Yang, M. S. Verma, J.-H. So, B. Mosadegh, C. Keplinger, B. Lee, F. Khashai, E. Lossner, Z. Suo, and G. M. Whitesides, "Buckling Pneumatic Linear Actuators Inspired by Muscle," Advanced Materials Technologies, vol. 1, no. 3, p. 1600055, Jun. 2016. [Online]. Available: https://onlinelibrary.wiley.com/doi/abs/10.1002/admt.201600055

[28] A. Hirsch and S. P. Lacour, "A Method to Form Smooth Films of Liquid Metal Supported by Elastomeric Substrate," Advanced Science, vol. 5, no. 10, p. 1800256, 2018. [Online]. Available: https://onlinelibrary.wiley.com/doi/abs/10.1002/advs.201800256 